Archives de sciences sociales des religions

159 | juillet-septembre 2012

Durkheim : Les formes élémentaires de la vie religieuse (1912)

\title{
Introduction : Des controverses aux héritages
}

\section{Pierre Lassave}

\section{(2) OpenEdition \\ Journals}

Édition électronique

URL : http://journals.openedition.org/assr/24666

DOI : $10.4000 /$ assr.24666

ISSN : $1777-5825$

Éditeur

Éditions de l'EHESS

Édition imprimée

Date de publication : 30 septembre 2012

Pagination : 9-16

ISBN : 978-2-7132-2330-3

ISSN : 0335-5985

Référence électronique

Pierre Lassave, "Introduction: Des controverses aux héritages », Archives de sciences sociales des religions [En ligne], 159 | juillet-septembre 2012, mis en ligne le 27 mars 2013, consulté le 21 avril 2019. URL : http://journals.openedition.org/assr/24666 


\section{Pierre Lassave}

\section{Introduction : Des controverses aux héritages}

Lorsque le premier numéro des Archives de sociologie des religions a vu le jour en 1956, Durkheim et la vie religieuse n'étaient plus au centre des sciences sociales en France. L'histoire de la sociologie rappelle aujourd'hui la petite phrase de Jean Stoetzel, l'un des plus influents refondateurs de la discipline après-guerre, reprochant à Durkheim d'avoir « inventé un phlogistique sociologique, stérile et paralysant » et se demandant s'il ne valait pas mieux "mettre les jeunes générations de futurs chercheurs à l'abri de son influence ». Mots durs prononcés en 1946 dans la faculté de Bordeaux où Durkheim a établi ses règles de la méthode et donné cent cinquante ans auparavant son premier cours de sociologie des religions. Malgré un tel verdict, Gabriel Le Bras, autre acteur influent de cette scène académique, a créé quelques années plus tard les Archives et le Groupe de sociologie des religions en s'inscrivant explicitement dans la filiation durkheimienne de L'Année sociologique. Les liens que le professeur d'histoire du droit canonique avait alors noués tant avec un monde universitaire en plein renouvellement qu'avec un monde catholique en proie à la crise des pratiques et des vocations sont sans doute à l'origine de ce coup de force. L'idée était alors de développer un champ d'études pour une sociologie en prise avec son temps, ici la sécularisation du monde, à l'instar des autres domaines thématiques du moment comme ceux de l'urbanisation, de l'industrialisation ou de la modernisation de la société. L'idée était aussi de renouer, pour ce faire, avec l'héritage durkheimien prolongé à l'entre-deux-guerres par l'Institut de sociologie dirigé par Marcel Mauss et dans lequel G. Le Bras fit ses premières armes en «morphologie religieuse » sous le regard bienveillant de Maurice Halbwachs.

Si les Règles de la méthode sociologique s'imposaient alors pour expliquer tout phénomène dit religieux par un ensemble déterminable d'autres faits sociaux, les Formes élémentaires de la vie religieuse (Formes désormais) n'ont d'abord été pour le groupe des Archives qu'une utile plateforme de définitions permettant par exemple de répartir les faits religieux entre les divers plans des communautés de croyance, des puissances surnaturelles et des contextes sociétaux. Comme s'il devait préserver sa démarche de connaissance de toute contamination croyante, G. Le Bras réservait le premier plan à la sociologie, le second relevant, selon lui, plutôt de la théologie et le troisième de l'histoire et de la science politique : une limitation du domaine qui est loin d'être allée de soi dans le premier noyau de chercheurs qu'il avait réunis autour de lui - Henri Desroche, François-André Isambert, Jacques Maître, Émile Poulat dont certains précisément s'étaient orientés vers la sociologie comme en une sorte de socioanalyse de leurs engagements ecclésiaux de jeunesse. On lira à ce propos l'entretien de Jacques Maître réalisé et commenté par Yann Potin, commentaire qui souligne par ailleurs les équivoques sémantiques, pour le moins, du passage de relais assuré par Le Bras.

Ouvrage classique aux ressources cognitives longtemps méconnues, les Formes n'ont pas moins aidé le groupe à ajuster son rapport à l'objet de connaissance et à trouver un langage commun pour le situer dans un continuum entre individu et société, foi et science, pensée dite primitive et pensée dite moderne. Outre ce service épistémologique, les Formes ont assuré plusieurs points de référence pour penser le monde contemporain, par exemple la théorie du sacré au moment des fameux retours de religiosité ici et ailleurs, ou la théorie de la morale quand il s'est agi de redéfinir les principes d'une laïcité adaptée à la globalisation du monde. Ce fut le double objet d'un numéro thématique de notre revue spécialement consacré à « Relire Durkheim » ( $n^{\circ}$ 69/1990), voilà déjà plus d'une vingtaine d'années. 
Dans la suite des précédentes commémorations, le centenaire du dernier ouvrage anthume de Durkheim incite à redécouvrir la richesse d'une théorie à l'aune des controverses et des prolongements qu'elle a suscités depuis lors. Tel est le parti éditorial pris par le présent numéro thématique qui s'attache à remonter le fil de la réception de l'œuvre depuis sa publication jusqu'aujourd'hui. Dans cette perspective, suivant un programme de recherche en Digital Humanities lancé par Stéphane Baciocchi ${ }^{1}$, un double corpus documentaire a été réuni dont on livre ici une première série d'analyses : d'une part, les nombreux comptes rendus qui ont accompagné la publication des Formes du vivant de leur auteur; d'autre part, une sélection d'articles, de notes critiques et de recensions qui dans notre propre revue ont directement traité des Formes au cours de ces cinquante dernières années. Ces deux corpus font l'objet d'une édition numérique sur le site de la revue ${ }^{2}$. Le premier représente plus de soixante-dix articles en différentes langues (l'ensemble totalise plus de huit cents feuillets), soit bien plus que la vingtaine de comptes rendus jusqu'ici commentés. Le second compte une trentaine de textes pour un volume d'environ deux cent cinquante pages. À noter qu'un troisième corpus est prévu pour l'année suivante, celui des références bibliographiques de plus de trois mille cinq cents livres ou articles recensés ou signalés dans la section de « sociologie religieuse » de L'Année sociologique, travail colossal de critique et de commentaire conduit par l'équipe durkheimienne de 1896 à 1913 qui fut comme l'on sait un véritable laboratoire pour la rédaction des Formes.

Les premières études que nous livrons à partir de ces documents nous plongent d'abord dans un moment explosif de rencontre entre une discipline savante et un de ses objets - la religion - des plus problématiques. Il n'allait pas de soi, en effet, de faire des croyances et des gestes les plus intimes et en même temps les plus discrètement réglés par des institutions multiséculaires un objet de connaissance réductible à ses constituants sociétaux. Sur fond de débats houleux autour de la séparation entre les Églises et l'État, transformer le dieu majuscule des monothéismes en la totalité sociétale ou historique qui l'a produit tout en allant chercher ses preuves dans l'électricité primitive des rituels totémiques ne pouvait qu'exacerber les antagonismes. Tout spécialement au sein d'une république des lettres aux idées déjà fortement ébranlées par les théories de l'évolution des espèces ou les conquêtes positives de la méthode expérimentale. En outre, la polyvalence constitutive des Formes, tout à la fois essai pionnier de définition sociologique de la religion, exploration des notions d'âme et d'esprit à travers les cultes totémiques et ensemble de prolégomènes à une théorie sociogénétique des catégories de l'entendement humain, a sans doute démultiplié les fronts et les objections.

Le spectre des réactions s'étend ainsi des revues les plus académiques aux magazines intellectuels les plus en vue. Se distinguent dans ce concert philosophes, anthropologues, théologiens et historiens, chaque polarité disciplinaire prolongeant ou contestant les définitions et hypothèses émises. Les historiens font alors parfois chorus avec les théologiens pour dénoncer le double procès en réduction consistant à faire passer le collectif devant l'individu en même temps que la forme élémentaire ou totémique devant la forme complexe ou chrétienne. Les anthropologues, anglo-saxons notamment, objectent à cet égard que les inadéquations variées entre le totem, le clan et les structures de la parenté ne permettent pas d'inférer une forme religieuse quelconque d'un totémisme abstrait. Les philosophes doutent de la solidité des maillons de la chaîne qui font remonter les notions de genre à la classe tribale,

1 Stéphane Baciocchi, François Théron, DD - Digital Durkheim 1912-2012. Présence et postérité des Formes élémentaires de la vie religieuse. Programme de recherche du laboratoire HASTEC, Paris, novembre 2011. Un carnet de recherche accompagne ce programme (http://digitaldurkheim.hypotheses.org)

$2 \mathrm{http}$ //assr.revues.org/22977 Le sommaire du premier corpus figure dans le document qui suit présenté par S. Baciocchi. Le sommaire du second corpus se retrouve en annexe de l'article de P. Lassave qui explique ses critères de sélection. 
de cause à la force du groupe, de temps au rythme des festivités saisonnières ou d'espace au territoire borné du clan ${ }^{3}$.

Au cœur de cette réception, dont les divers fronts ont déjà fait l'objet de premières études rétrospectives depuis quelques années ${ }^{4}$, les intellectuels catholiques se sont particulièrement distingués par leurs critiques acerbes. Leurs objections multiples de méthode et de fond ont porté tant sur la réduction de l'esprit à une force collective que sur l'incommensurabilité entre des formes élémentaires mais abstraites et des formes réelles mais plus complexes. Une fin de non-recevoir sans doute due au caractère alors très contre-intuitif du point de vue sociologique pour des croyants et à sa prétention hégémonique, sans parler de son prophétisme latent (voir la contribution de Guillaume Cuchet). La division des « deux France » avec l'Affaire Dreyfus et la question de la laïcisation de l'enseignement ainsi que les tensions de la crise moderniste au sein du magistère catholique ne sont pas étrangères aux tournures les plus polémiques enveloppant le débat de fond sur la naissance d'une école de sociologie religieuse. Fils d'une lignée de rabbins, Durkheim a pu ainsi être accusé d'avoir inventé une « science juive ». Outre l'antisémitisme, la passion nationaliste a également reproché au sociologue, bon connaisseur des sciences sociales allemandes, d'être allé chercher son inspiration Outre-Rhin.

La réception première des Formes dans ce pays est plus importante qu'on ne le croit. Une traduction allemande était en projet avant même que la première traduction anglo-américaine de Joseph Ward Swain ne trouve un éditeur. Abandonné, sans doute avec le déclenchement de la guerre, ce projet de traduction et les premières recensions allemandes montrent en tout cas que l'ouvrage n'était pas méconnu des ethnologues engagés dans le débat totémique et des historiens intéressés par une perspective sociogénétique sur l'origine du sentiment religieux. Aucune référence n'est cependant faite à l'ouvrage dans les textes de Max Weber sur la sociologie des religions, ce qui laisse supposer que celui-ci ne le connut pas. Au-delà de cette première réception, plutôt partielle, il faudrait sans doute poursuivre l'examen de la pénétration progressive de l'école durkheimienne dans les universités allemandes jusqu'au coup d'arrêt momentané provoqué par la montée du nazisme. Avant ce terme fatal, le Père Wilhelm Schmidt, fondateur de l'école viennoise d'ethnologie dans les années 1910-1930, ne s'est pas privé de relayer diverses critiques en stigmatisant la «théorie pantotémiste » de Durkheim (voir Michael Löwy).

En Italie, c'est surtout dans la Rivista Italiana di Sociologia, lieu central de la sociologie naissante dans ce pays au tournant $\mathrm{du} \mathrm{Xx}^{\mathrm{e}}$ siècle, que les thèses de Durkheim ont été publiées, commentées et discutées. Quelques disciples fervents comme Alessandro Bruno n'ont pourtant pas totalement convaincu de leur pertinence et de leur nouveauté au sein d'une communauté savante qui restera longtemps sous l'influence du paradigme évolutionniste anglo-saxon et de la pensée logiciste, libérale et pessimiste de Vilfredo Pareto (Salvatore Abbruzzese).

Pour revenir en France, le second temps de réception des Formes, qui court depuis la refondation de la sociologie au lendemain de la Seconde Guerre mondiale, est naturellement plus actuel mais aussi plus « refroidi » que le premier moment. Il s'agit d'ailleurs de lectures partielles et discontinues, qui font la postérité variable d'un classique, plutôt que d'une réception au sens fort du terme. Ce temps contemporain n'est abordé ici qu'à travers le corpus des Archives tel que nous l'avons présenté, lequel aussi restreint soit-il fait cependant écho

3 Voir Stéphane Baciocchi, « Origine de la religion/Religion des primitifs. La Première réception des Formes élémentaires de la vie religieuse, 1912-1917», Université Montesquieu - Bordeaux IV, Communication au colloque international «Les Formes élémentaires de la vie religieuse de Durkheim cent ans après (1912-2012)», 11 juin 2012.

4 Cf. Steven Lukes, Émile Durkheim, His Life and Work: A Historical and Critical Study, Londres, Penguin Press, 1992 [1 $1^{\text {st }}$ edit. 1973]; William S. Pickering, Durkheim on Religion. A Selection of Readings with Bibliographies and Introductory Remarks, Londres, James Clarke \& Co Ltd, 2011 [1 $1^{\text {st }}$ edit. 1973]; Durkheim's Sociology of Religion: Themes and Theories, Londres, James Clarke \& Co Ltd., 2009 [1 ${ }^{\text {st }}$ edit. 1984]. 
aux transformations de perspectives et de paradigmes dans les disciplines (Pierre Lassave). La désormais célèbre phrase de Durkheim commentant son livre devant une assemblée de libres penseurs, selon laquelle quiconque étudierait la religion sans partager le sentiment de ses adeptes " ressemblerait à un aveugle qui parlerait de couleurs", fait ainsi point de repère. Elle fut d'abord exhumée par les Archives, peu avant le moment de retour aux textes de Durkheim qui depuis les années 1970 n'a cessé de s'amplifier avec les Durkheimian Studies. Elle fut aussi reprise comme un slogan pour élargir le champ de vision du sociologue à celui de l'expérience ethnographique des croyances qu'il objective en même temps qu'elles le traversent, pour se départir donc des limitations d'objet imposées par une vision restrictive de l'héritage durkheimien, et pour remettre en cause l'image strictement positiviste attachée à l'appréhension des faits sociaux comme choses.

À ce débat général autour de la rupture ou de la continuité entre l'objet et le sujet de connaissance correspond pour la sociologie des religions la remise en chantier des thèses de Durkheim. Aidé par la redécouverte de textes que l'histoire aronienne de la pensée sociologique avait délaissés à l'archiviste, François-André Isambert a ainsi progressivement établi dans une approche synchronique les règles principales d'une grammaire du sacré et de la morale. Avec pour chacune de ces notions : un indice (l'interdit pour le sacré et la sanction pour la morale) ; une structure duale (division entre le sacré et le profane pour l'un et entre esprit de discipline et attachement au groupe pour l'autre); un jeu à trois (l'ambivalence naturelle du sacré pour l'un et le dépassement des obligations par autonomie de la volonté pour l'autre) ; une régulation (par le rite pour l'un, par l'incorporation créatrice de valeurs pour l'autre). Soit une grille de lecture disponible parmi d'autres pour confronter les traditions entre elles, qu'elles relèvent des Églises ou des États.

De là s'est ouvert, à l'approche de la fin du siècle, un chantier transversal sur la philosophie du croire pour lequel la relecture des Formes a pu être utile. Raymond Boudon par exemple, président d'honneur de L'Année sociologique, retrouve dans les Formes une véritable axiomatique déployée autour de la différenciation des systèmes de connaissances et de leur transférabilité relative: ce qui était prodige positif pour les antiques devient miracle extraordinaire pour les modernes. Boudon voit surtout dans ce « chef-d'œuvre » sociologique confirmation de sa propre théorie de la rationalité ordinaire selon laquelle les causes ultimes $\mathrm{du}$ fait qu'on croit à ce qu'on croit résident dans les raisons qu'on a d'y croire. Autant donc de reprises conceptuelles des Formes qui dépassent depuis lors largement l'objet religieux propre aux Archives.

Au-delà de ces dernières et de leur corpus, les Formes ont rencontré des fortunes diverses que l'on n'est pas près de circonscrire. Un regard porté sur la Chine indique ainsi que si l'ouvrage fut tardivement traduit, il n'en constitue pas moins un jalon dans l'histoire universitaire de la discipline : dans les années 1920, il figura en bonne place comme un des modèles pour la discipline naissante; dans les années d'après la révolution culturelle, il s'imposa comme référence pour la reconstruction du savoir; dans les années actuelles de retour aux traditions, il représente un classique appelant traduction et commentaire critique dans un immense pays où la religion se distingue difficilement des rites de la vie ordinaire (Laurence Roulleau-Berger, Liu Zhengai).

C'est parfois d'ailleurs par l'intermédiaire de lectures secondaires que l'œuvre durkheimienne prend toute sa portée historique ; par exemple en Haïti dans les années 1930, l'essai remarqué Ainsi parla l'Oncle (1928) de Jean Price-Mars, écrivain et homme d'État haïtien mais aussi ethnologue occasionnel formé par Mauss. Cet essai précurseur des théories de la négritude et de la créolité contribua grandement à dépénaliser le culte vodou dans le pays et à en faire, via Durkheim, une forme évoluée de la vie religieuse locale (Lewis Ampidu Clorméus). 
Structuré autour de l'étude des deux corpus de textes réunis, notre dossier les dépasse donc largement. S'intercalent ainsi entre leurs commentaires respectifs des coups de projecteur sur la matière même des objets traités dans les Formes.

Il en est ainsi de l'édition de notes de cours donnés dans les années 1920 par Marcel Mauss à l'École pratique des hautes études; notes sur le rituel festif du corrobori australien accompagnées d'une réflexion autour de la manière dont Mauss reprend la question de son oncle et maître sur l'effervescence collective en ouvrant de multiples perspectives autour des notions de rythme et d'influx nerveux (Jean-François Bert).

Il en va également des churinga australiens, ces objets dévotionnels des peuples aborigènes, morceaux de bois ou de pierre dessinés aux formes oblongues que l'on fait vibrer dans l'air les jours de sortie et que l'on préserve des regards le reste du temps pour ne pas troubler l'esprit de l'ancêtre qui les habite. Claude Lévi-Strauss voyait en eux des archétypes lointains des documents d'archives d'aujourd'hui, objets sacralisés précieusement conservés et parcimonieusement exhibés. Il faut sans doute passer des churinga australiens aux fétiches africains pour se convaincre de la choséité des esprits ou des dieux dont l'objet de vénération est témoin (André Mary). Du sang sacrificiel grâce auquel tout le clan se reconnaît à l'appropriation intime de ses idoles coagulées, la représentation de soi passe d'un objet transitionnel à d'autres. Le débat sur la représentation des choses sacrées lancé par Durkheim se serait-il déplacé ainsi sur le terrain de la tension entre réification et médiation?

Il en est également ainsi du temps, catégorie centrale de la mentalité humaine dont les Formes entreprennent la sociogenèse. Dans ses travaux sur les cadres sociaux de la mémoire, Maurice Halbwachs, élève de Durkheim, se demande ainsi si la notion de temps existe encore dans le rêve ou si elle disparaît dans les profondeurs du moi onirique comme l'affirment certains psychologues. À l'encontre de ces derniers, il souligne la prégnance du temps impersonnel comme moyen d'existence du rêve dans la mémoire, confirmation des thèses durkheimiennes qui ne valident pas pour autant les conclusions sociogénétiques du maître (Thomas Hirsch).

Il en est ainsi enfin de l'instance économique, à première vue absente du Durkheim des Formes. Absence pourtant toute relative ainsi qu'une lecture attentive le révèle à travers l'opposition entre le culte comme source première de cohésion sociale et l'échange marchand comme lieu de l'incohésion sinon de l'anomie (Philippe Steiner). De la Division du travail social aux Formes, Durkheim a en fait délaissé la dimension institutionnelle du marché, malgré son collaborateur François Simiand qui voyait dans la monnaie une institution sociale centrale. Ce contraste est aujourd'hui encore plus visible lorsqu'on met en perspective les reconnaissances successives de l'économique comme institution, y compris dans ses rapports aux faits religieux. Par exemple, l'ascétisme puritain comme schème opérateur du lien entre le monde humain et le salut éternel tel que Max Weber l'a développé ; et plus récemment encore, l'institution marchande comme dispositif-cadre du gouvernement des hommes par les choses, comme Michel Foucault a pu l'esquisser.

Centré sur la postérité longue et variable des Formes, en ses moments de réception vivante, d'embaumement chez les classiques et de retour en grâce de référence légitimatrice, notre dossier ne peut prétendre à un improbable bilan. L'exploration des usages divers d'un essai centenaire n'a pas fini de nous révéler la subtilité de ses filiations et filières conceptuelles, la continuité de ses reprises académiques et l'actualité de ses effets politiques. Nul doute que l'attention naturellement portée à la vie religieuse ou à la chose sacrée laisse un peu dans l'ombre les multiples inflexions données depuis quelques années par les sciences cognitives à la sociogenèse durkheimienne des catégories de l'entendement. La matrice sacrée à l'origine de toute classification des éléments du monde pourrait bien par exemple devoir composer avec la sélection naturelle des «mèmes », ces unités d'information réplicatives d'une culture à l'autre. Mais les quelques cas d'usages ici réunis convergent sur la vitalité paradoxale d'un 
objet aussi problématique que celui que la modernité occidentale a désigné du mot de religion. Il suffit d'éloigner le regard pour voir, par exemple, qu'en Chine le mot trouve toute sa place académique et institutionnelle tout en se distinguant très peu d'une ritualité quotidienne qui se passe pratiquement fort bien de lui ; sans parler de la légitimation politique que les Formes ont indirectement conférée à un vodou haïtien qui sort de sa nuit esclave. Les fenêtres entrebâillées sur le rôle joué par l'œuvre durkheimienne en Allemagne et en Italie mériteraient d'être largement ouvertes comme le suggère la question des liens entre l'ethnologie, la mission chrétienne et la théologie. On n'a fait qu'entrevoir ici comment la théorie du monothéisme primitif prônée par l'École viennoise d'ethnologie religieuse du Père Schmidt a su trouver ses relais de terrain chez les Pygmées d'Afrique équatoriale pour faire pièce à l'évolutionnisme anglo-saxon ou au sociologisme durkheimien. Et le coup de projecteur donné sur la Rivista Italiana di Sociologia en appelle sans doute d'autres. L'enquête internationale et interdisciplinaire attend donc d'être poursuivie. Elle pourrait même investir le présent d'autres lieux épistémiques, ne serait-ce que pour comprendre, par exemple, comment la durkheimologie s'exprime depuis quelques années en langue anglaise. Gageons que les références bibliographiques dont la liste figure dans le document qui suit et que le site internet de notre revue met en libre accès saura être le tremplin de nouvelles découvertes.

Pierre LASSAVE

Centre d'Études Interdisciplinaires des Faits Religieux (EHESS-CNRS) pierre.lassave@orange.fr 